\title{
The cross-section data from neutron activation experiments on niobium in the NPI p-7Li quasi-monoenergetic neutron field
}

\author{
M. Honusek ${ }^{1, a}$, P. Bém ${ }^{1}$, U. Fischer ${ }^{2}$, M. Götz ${ }^{1}$, J. Novák ${ }^{1}$, S.P. Simakov² ${ }^{2}$ and E. Šimečkováa ${ }^{1}$ \\ ${ }^{1}$ Nuclear Physics Institute, CZ-25068 Řež, Czech Republic \\ 2 Karlsruhe Institute of Technology, KIT INR, 76021 Karlsruhe, Germany
}

\begin{abstract}
The reaction of protons on ${ }^{7} \mathrm{Li}$ target produces the high-energy quasimonoenergetic neutron spectrum with the tail to lower energies. Proton energies of 19.8, $25.1,27.6,30.1,32.6,35.0$ and $37.4 \mathrm{MeV}$ were used to obtain quasi-monoenergetic neutrons with energies of $18,21.6,24.8,27.6,30.3,32.9$ and $35.6 \mathrm{MeV}$, respectively. $\mathrm{Nb}$ cross-section data for neutron energies higher than $22.5 \mathrm{MeV}$ do not exist in the literature. $\mathrm{Nb}$ is the important material for fusion applications (IFMIF) as well. The variable-energy proton beam of NPI cyclotron is utilized for the production of neutron field using thin lithium target. The carbon backing serves as the beam stopper. The system permits to produce neutron flux density about $10^{9} \mathrm{n} / \mathrm{cm}^{2} / \mathrm{s}$ in peak at $30 \mathrm{MeV}$ neutron energy. The niobium foils of $15 \mathrm{~mm}$ in diameter and approx. $0.75 \mathrm{~g}$ weight were activated. The nuclear spectroscopy methods with HPGe detector technique were used to obtain the activities of produced isotopes. The large set of neutron energies used in the experiment allows us to make the complex study of the cross-section values. The reactions $(n, 2 n),(n, 3 n),(n, 4 n)$, $(\mathrm{n}, \mathrm{He} 3),(\mathrm{n}, \alpha)$ and $(\mathrm{n}, 2 \mathrm{n} \alpha)$ are studied. The cross-sections data of the $(\mathrm{n}, 4 \mathrm{n})$ and $(\mathrm{n}, 2 \mathrm{n} \alpha)$ are obtained for the first time. The cross-sections of $(n, 2 n)$ and $(n, \alpha)$ reactions for higher neutron energies are strongly influenced by low energy tail of neutron spectra. This effect is discussed. The results are compared with the EAF-2007 library.
\end{abstract}

\section{Introduction}

Neutron generator based on $\mathrm{p}-{ }^{7} \mathrm{Li}$ reaction at NPI Rez allows us to study cross-section data using quasimonoenergetic neutron spectra up to $35 \mathrm{MeV}$ neutron energy. Rez apparatus is described in details in ref. [1]. Therefore we are able to measure cross-section data at incident neutron energies higher than $20 \mathrm{MeV}$, where such data are scarce. Such data are needed for various technological applications like IFMIF as well. We measured our data for 7 proton beam energies and therefore we are able to analyse cross-section in the complexity. Two sets of different neutron spectra are used in the analysis: measured by TOF methods [2] and simulated. The results are compared with cross section data from EAF-2007 library [3].

\section{Experimental equipment}

The overall view of the target station for p-Li experiments is shown in Fig. 1 (left). The detail view of the reaction chamber is shown in Fig. 1 (right). Irradiated $\mathrm{Nb}$ foils are placed on the right side of the apparatus at distances 48 and $88 \mathrm{~mm}$ from the Li foil.

\footnotetext{
a e-mail: honusek@ujf.cas.cz
}

This is an Open Access article distributed under the terms of the Creative Commons Attribution-Noncommercial License 3.0, which permits unrestricted use, distribution, and reproduction in any noncommercial medium, provided the original work is properly cited. 

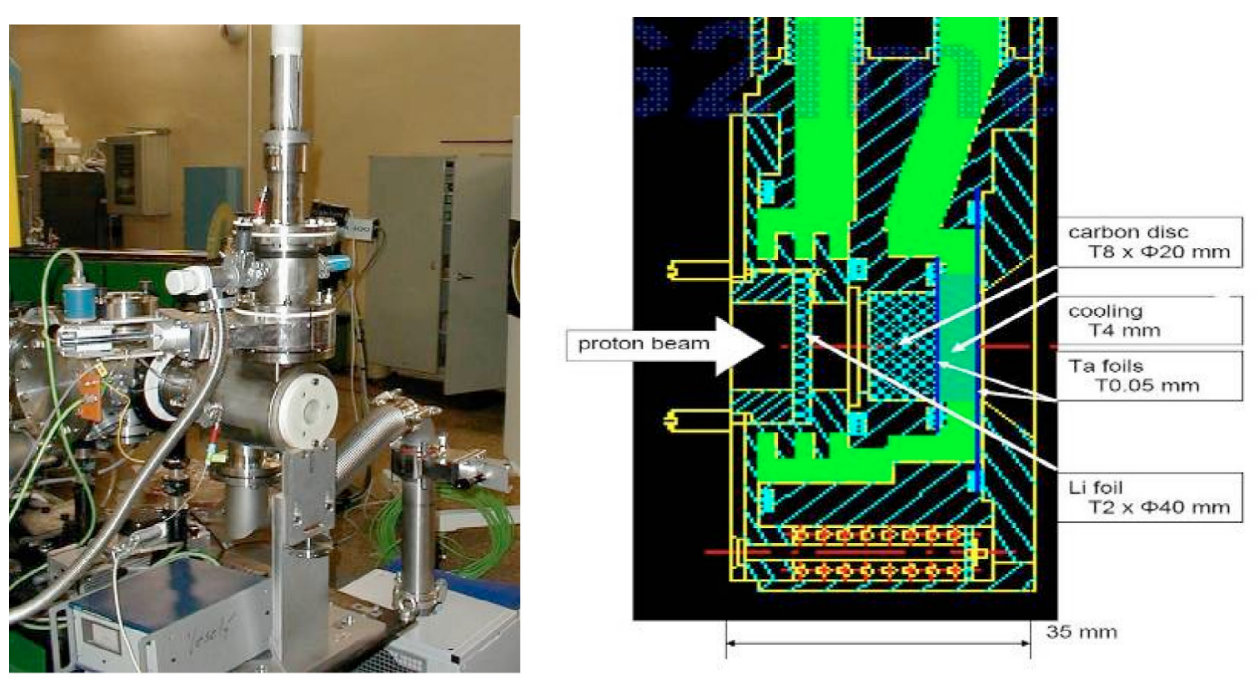

Fig. 1. The overall view to the target station of NPI p- ${ }^{7} \mathrm{Li}$ neutron source (left). The detail view of the reaction chamber (right).

Niobium foils diam. $15 \mathrm{~mm}$ and weights approx. $0.75 \mathrm{~g}$ were irradiated at the distances of 48 and $88 \mathrm{~mm}$ from the lithium foil. The typical proton beam current was $3 \mu \mathrm{A}$, typical time of irradiation (at one proton beam energy run) was $20 \mathrm{~h}$. The time profile of the proton beam current was monitored. Proton beam energies of 19.8, 25.1, 27.6, 30.1, 32.6, 35.0 and $37.4 \mathrm{MeV}$ were used at 7 experimental runs.

Irradiated samples were investigated by means of gamma spectroscopy methods. Two HPGe detectors of 23 and $50 \%$ efficiencies and FWHM of $1.8 \mathrm{keV}$ at $1.33 \mathrm{MeV}$ were used. Activated isotopes were identified on the basis of half-lifes, gamma ray energies and intensities. Experimental results RR were obtained as number of produced atoms $/ 1 \mu \mathrm{A}$ of proton beam $/ 1 \mathrm{~kg}$ of mass of target.

\section{Neutron spectra}

Neutron spectra consist of quasi-monoenergetic part corresponding to the reactions to g.s. and $0.429 \mathrm{MeV}$ state in ${ }^{7} \mathrm{Be}$, low-energy tail generated a) by reactions on ${ }^{7} \mathrm{Li}$ leading to further excited states in ${ }^{7} \mathrm{Be}$ and other reactions on ${ }^{7} \mathrm{Li}$ and $\mathrm{b}$ ) by reactions of protons on carbon stopper.

Parameter RR is simply connected with the product of neutron spectrum NS(E) and cross section $\mathrm{CS}(\mathrm{E}): \mathrm{RR} \sim \int \mathrm{NS}(\mathrm{E}) \times \mathrm{CS}(\mathrm{E}) \mathrm{dE} \sim \sum \mathrm{NS}(\mathrm{E}) \times \mathrm{CS}(\mathrm{E}) \Delta \mathrm{E}$. The corresponding set of equations is to be solved.

Two sets of neutron spectra were used in our evaluation.

- Spectra measured using TOF by Y.Uwamino et al. [2] at long distance (the neutron spectra for the proton energies not included in [2] were calculated according procedure described in ref. [4]). The example of the spectrum is shown in Fig. 2. We used closer geometry and therefore e.g. different distances of $\mathrm{Li}$ and $\mathrm{C}$ foils and angular dependency are not included in such an analysis.

- Spectra simulated [5], experimental conditions (Li foil, thick C beam stopper, alcohol coolant, flanges, experimental hall) included. For the simulation at both distances MCNPX and LA-150h proton cross section library were used. Spectra used in our analysis for geometry $88 \mathrm{~mm}$ (distance $\mathrm{Li}$ foil - Nb foil) are shown in Fig. 3.

\section{Data analysis}

The observed reactions are listed in Table 1 . Isotope $\mathrm{Nb93}$ is only one stable isotope of $\mathrm{Nb}$. 


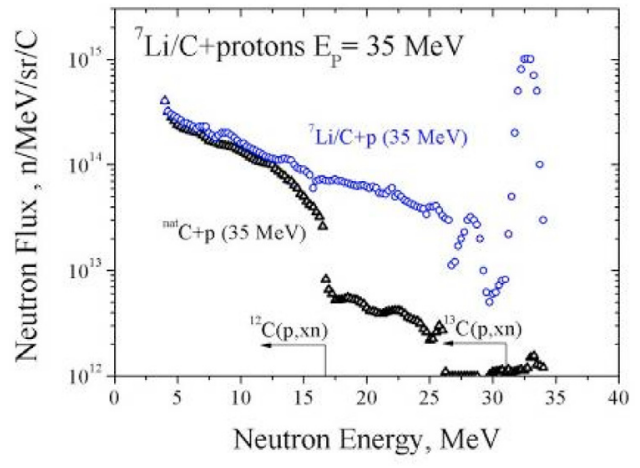

Fig. 2. Typical neutron spectrum (TOF) [2].

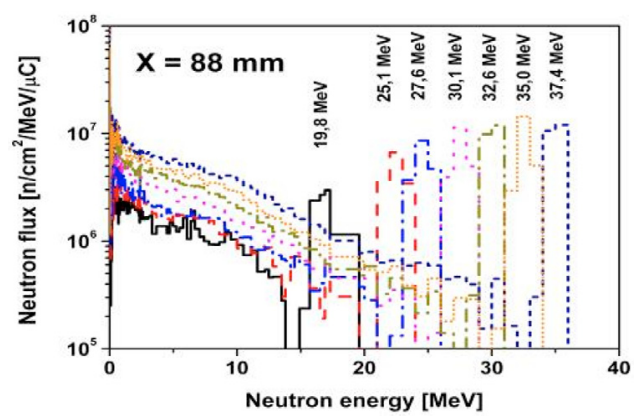

Fig. 3. Neutron spectra (simulated) [5].

Table 1. Isotopes observed from irradiation of $\mathrm{Nb}$ foils.

\begin{tabular}{lccc}
\hline Isotope & T1/2 & reaction & threshold $(\mathrm{MeV})$ \\
\hline Nb90 & $14.60 \mathrm{~h}$ & $(\mathrm{n}, 4 \mathrm{n})$ & 29.078 \\
$\mathrm{Nb} 91 \mathrm{~m}$ & $60.86 \mathrm{~d}$ & $(\mathrm{n}, 3 \mathrm{n})$ & 16.999 \\
$\mathrm{Nb92m}$ & $10.15 \mathrm{~d}$ & $(\mathrm{n}, 2 \mathrm{n})$ & 9.063 \\
Y91m & $49.71 \mathrm{~m}$ & $(\mathrm{n}, 3 \mathrm{He})$ & 8.362 \\
Y90m & $3.19 \mathrm{~h}$ & $(\mathrm{n}, \alpha)$ & 0 \\
Y88 & $106.65 \mathrm{~d}$ & $(\mathrm{n}, \alpha 2 \mathrm{n})$ & 13.554 \\
\hline
\end{tabular}

\subsection{Error analysis}

Only the gamma spectroscopy errors ( $\mathrm{min} .3 \%$ ) are used in the next part of the discussion of the results. The systematical errors of our beam current measurement $(5 \%)$ and the error of beam current measurement of Y. Uwamino [2] as $10 \%$ should be added to gamma spectroscopy errors. The uncertainty of MCNPX is estimated as $10 \%$ for quasi-monoenergetic neutrons.

\section{Experimental results}

The experimental cross sections CS as a function of neutron energy En are shown in next figures. Our experimental data are presented for both irradiation distances 48 and $88 \mathrm{~mm}$ of $\mathrm{Nb}$ foils from the Li foil. In each case the different neutron spectra (see previous part of article) are used in the analysis, Y. Uwamino (Uw TOF) [2] and simulated (MCNPX) [5].

EAF-2007 library cross sections are shown as well; the proper feeding of the studied nuclei and possible other reaction channels (e.g. $\alpha+$ ) are included. EXFOR data are shown as well.

We have to subtract low energy bump of cross sections lower $\sim 20 \mathrm{MeV}$ in two reactions ((n,2n) and $(\mathrm{n}, \alpha))$. For this purposes we use EAF-2007 library [3]. Firstly we will discuss these reactions. Subtraction of this bump in other reaction does not strongly influence the results.

\subsection{Reaction $(n, 2 n)$}

The activation cross-sections of isomeric state ${ }^{92 \mathrm{~m}} \mathrm{Nb}$ were measured. The spectrum tail to lower energies below our experimental points is subtracted using EAF-2007 cross section library. Error of subtraction procedure as 5\% is accepted. The high energy bump in the case of UwTOF spectra disappear when real experimental geometry (MCNPX) is taken into account. The data are close to EAF-2007 library. See Fig. 4. 


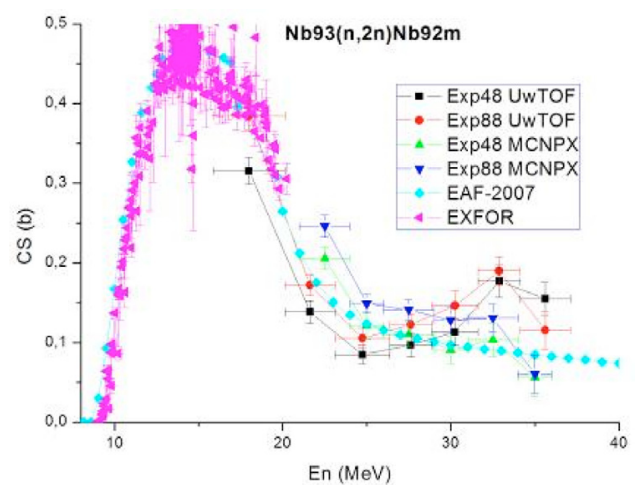

Fig. 4. Reaction ${ }^{93} \mathrm{Nb}(\mathrm{n}, 2 \mathrm{n}){ }^{92 \mathrm{~m}} \mathrm{Nb}$.

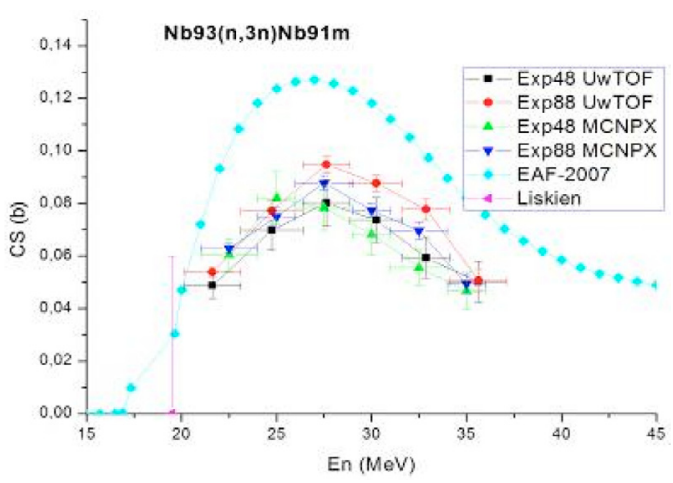

Fig. 6. Reaction ${ }^{93} \mathrm{Nb}(\mathrm{n}, 3 \mathrm{n}){ }^{91 \mathrm{~m}} \mathrm{Nb}$.

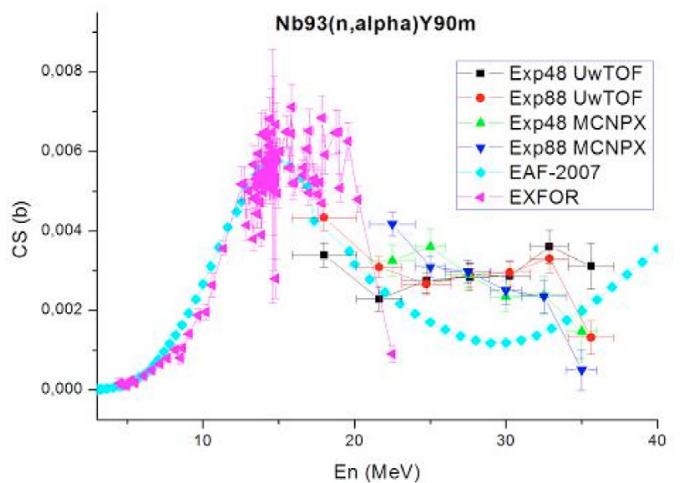

Fig. 5. Reaction ${ }^{93} \mathrm{Nb}(\mathrm{n}, \alpha)^{90 \mathrm{~m}} \mathrm{Y}$.

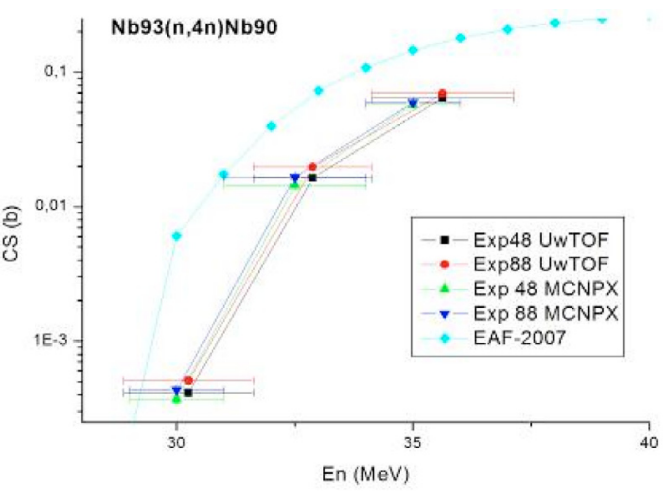

Fig. 7. Reaction ${ }^{93} \mathrm{Nb}(\mathrm{n}, 4 \mathrm{n}){ }^{90} \mathrm{Nb}$.

\subsection{Reaction $(\mathrm{n}, \alpha)$}

The activation cross-sections of isomeric state ${ }^{90 \mathrm{~m}} \mathrm{Y}$ were measured. Similarly to the previous reaction the low energy part is subtracted using EAF-2007 data, error of 10\% is accepted. The behaviour of the MCNPX data was correctly described in $(n, 2 n)$ reaction. We may come to the conclusion that EAF-2007 database does not correctly describe the reaction ${ }^{93} \mathrm{Nb}(\mathrm{n}, \alpha)^{90 \mathrm{~m}}$ Y. See Fig 5.

\subsection{Other reactions}

Reaction (n,3n) activated isomeric state ${ }^{91 \mathrm{~m}} \mathrm{Nb}$ (Fig. 6), reaction (n,4n) activated ground state ${ }^{90} \mathrm{Nb}$ (Fig. 7), reaction $\left(\mathrm{n},{ }^{3} \mathrm{He}\right)$ activated isomeric state ${ }^{91 \mathrm{~m}} \mathrm{Y}$ (Fig. 8) and reaction (n, $\left.\alpha 2 \mathrm{n}\right)$ activated ground state ${ }^{88}$ Y (Fig. 9).

The EAF-2007 data are substantially higher than the experimental ones in the reactions (n,3n), $(\mathrm{n}, 4 \mathrm{n})$ and $(\mathrm{n}, 3 \mathrm{He})$. The data of $(\mathrm{n}, \alpha 2 \mathrm{n})$ reaction are described properly using EAF-2007.

The energy intervals used in VITAMIN $\mathrm{J}+$ for higher energies are too large for cross section calculations and MCNPX simulations. 
EFNUDAT Workshop “Measurements and Models of Nuclear Reactions", Paris, 2010

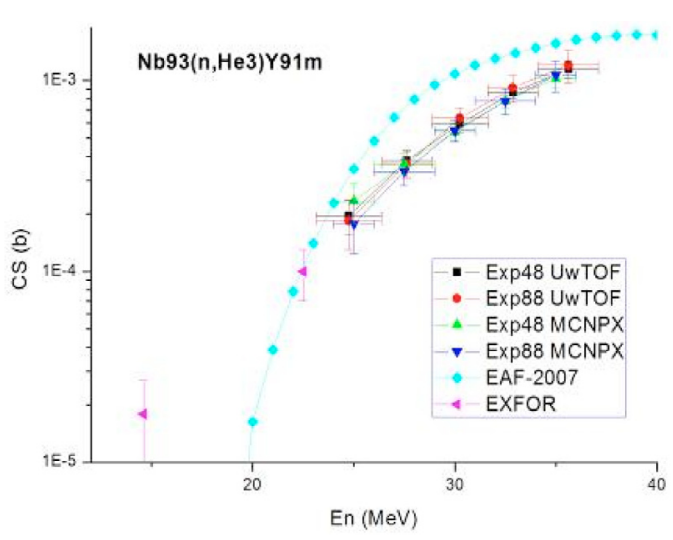

Fig. 8. Reaction ${ }^{93} \mathrm{Nb}\left(\mathrm{n},{ }^{3} \mathrm{He}\right){ }^{91 \mathrm{~m}} \mathrm{Y}$.

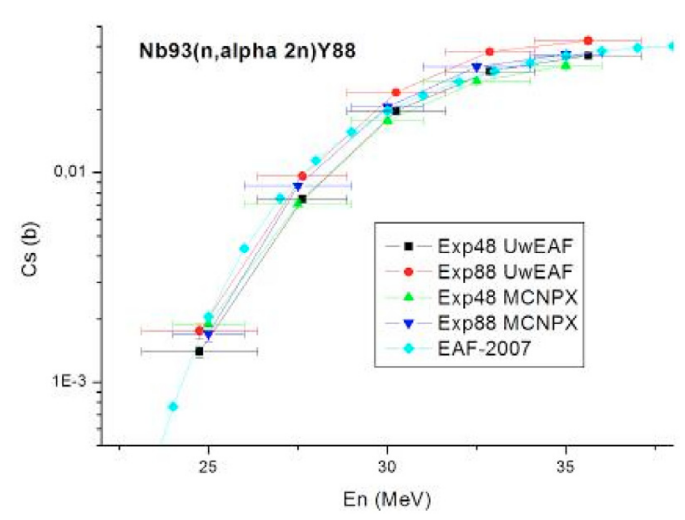

Fig. 9. Reaction ${ }^{93} \mathrm{Nb}(\mathrm{n}, \alpha 2 \mathrm{n})^{88} \mathrm{Y}$.

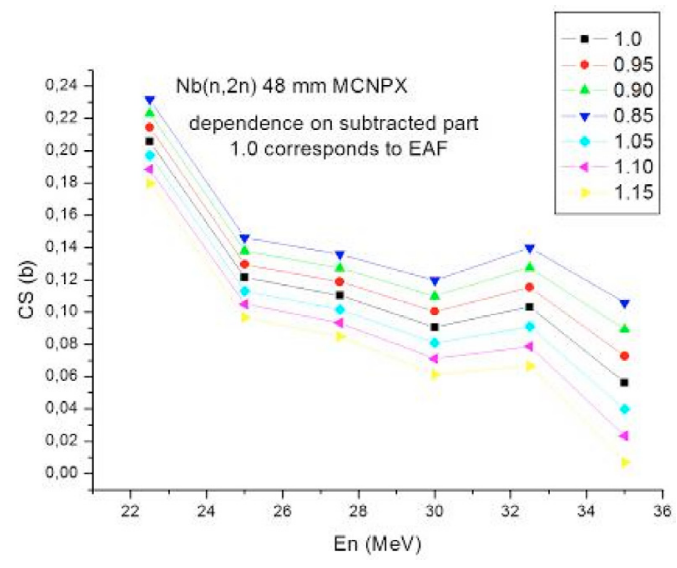

Fig. 10. Dependence of CS data on multiplications coefficients, reaction ${ }^{93} \mathrm{Nb}(\mathrm{n}, 2 \mathrm{n})^{92 \mathrm{~m}} \mathrm{Nb}$.

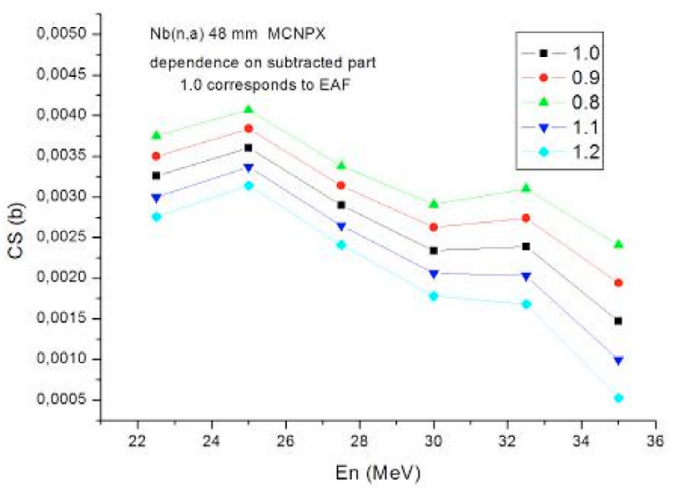

Fig. 11. Dependence of CS data on multiplications coefficients, reaction ${ }^{93} \mathrm{Nb}(\mathrm{n}, \alpha){ }^{90 \mathrm{~m}} \mathrm{Y}$.

\section{Errors and correlations - some comments}

\subsection{The influence of cross-section low energy bump}

We will discuss the effect of low-energy cross-section bump (neutron energies $<\sim 20 \mathrm{MeV}$ ) on our data in more details (reactions $(\mathrm{n}, 2 \mathrm{n})$ and $(\mathrm{n}, \alpha)$ ). For this purposes we introduce the coefficient $\mathrm{M}$ which multiply EAF data in this region ( $\mathrm{M}=1$ means EAF-2007 library). Examples of such analyses are shown in Figs. 10, 11 for the reactions $(n, 2 n)$ and $(n, \alpha)$, respectively.

The errors of these data are not shown due to complexity of the pictures. The difference between CS data for $M=1$ and $C S$ data for other values $M$ are within errors or close to the value.

\subsection{The effect of correlation coefficients}

The set of equations presented in section 3 is written as the matrix $R R=A^{*} C S$. Solving this equation we introduce correlations between CS. Let we introduce weight matrix $W$, where only diagonal elements are non zero and equals reciprocal squares of errors of RR. Then error matrix of CS is $\left(A^{\prime} W A\right)^{-1}$. 
Analysing the obtained data we come to the conclusion that the effect of correlations is rather small in our case.

\section{Conclusions}

Quasi-monoenergetic neutron production based on $\mathrm{p}-{ }^{7} \mathrm{Li}$ source is used to study high energy neutron reactions on ${ }^{93} \mathrm{Nb}$ target. New cross section data for energy of neutrons higher than $23 \mathrm{MeV}$ on niobium for the reactions $(n, 2 n),(n, 3 n),(n, 4 n),(n, 3 H e),(n, \alpha)$ and $(n, \alpha 2 n)$ are presented. These data are needed for technological applications like IFMIF and serve as the test of neutron databases and nuclear models as well. Experimental cross sections are obtained for the distances 48 and $88 \mathrm{~mm}$ of irradiated foils from Li foil for both neutron spectra: measured TOF and simulated MCNPX.

Taking into account the fact that MCNPX simulation describes properly the cross section behaviour in $(n, 2 n)$ reactions we may suppose that EAF-2007 database (including possible reaction channels) does not correctly describe experimental data in $(n, \alpha+)$ reaction.

EAF-2007 data overestimate evidently the experimental ones in reactions (n,3n), (n,4n) and (n,3He). The data of (n, $\alpha 2 \mathrm{n})$ reaction are described properly.

The gamma spectroscopy errors only are shown in our work. Systematical lowering of about 10-20\% of CS data at $48 \mathrm{~mm}$ comparing to CS data a $88 \mathrm{~mm}$ geometry have to be studied in future. We came to the conclusion that the energy intervals in VITAMIN J+ structure for higher energy regions are too large for both the cross section presentation and MCNPX simulations.

This work was supported by Fusion for Energy under the grant contract No. F4E-2008-GRT-014 (ES-AC). The views and opinions expressed herein reflect only the author's views. Fusion for Energy is not liable for any use that may be made of the information contained therein.

The authors are indebted to the operating crew of the U-120M cyclotron for the ready assistance.

\section{References}

1. P. Bém, V. Burjan, J. Dobeš, U. Fischer, M. Götz, M. Honusek, V. Kroha, J. Novák, S.P. Simakov, and E. Šimečková, "The NPI cyclotron-based fast neutron facility," Proc. Int. Conf. on Nuclear Data for Science and Technology (ND 2007), p.555, Nice, France, April 22-27, 2007.

2. Y. Uwamino, T.S. Soewarsono, H. Sugita, Y. Uno, T. Najamura, T. Shibata, M. Imamura, S. Shibata, "High-energy p-Li neutron field for activation experiment," Nucl. Instr. Meth. A, 389, 463 (1997).

3. R.A. Forrest, J. Kopecky, M. Pillon, A. Klix, S.P. Simakov, J-Ch. Sublet, P. Bém, M. Honusek and E. Šimečková, "Validation of EASY-2007 using integral measurements" UKAEA FUS 547, UKAEA 2008, United Kingdom.

4. P. Bém, V. Burjan, U. Fischer, M. Götz, M. Honusek, V. Kroha, J. Novák, S.P. Simakov, and E. Šimečková, "Neutron activation experiments on chromium and tantalum in the NPI p-7Li quasimonoenergetic neutron field," Proc. Int. Conf. on Nuclear Data for Science and Technology (ND 2007), p. 983, Nice, France, April 22-27, 2007.

5. S.P. Simakov, P. Bém, V. Burjan, U. Fischer, R.A. Forrest, M. Götz, M. Honusek, V. Kroha, J. Novák and E. Šimečková, "Analysis of the dosimetry cross sections measurements up to $35 \mathrm{MeV}$ with a ${ }^{7} \mathrm{Li}(\mathrm{p}, \mathrm{xn})$ quasi-monoenergetic neutron source“, Int. Conf. on Nuclear Data for Science and Technology (ND 2010), Jeju Island, Korea, April 26-30 (2010). 